\title{
WELL-BALANCED ROE-TYPE NUMERICAL SCHEME FOR A MODEL OF TWO-PHASE COMPRESSIBLE FLOWS
}

\author{
Mai Duc Thanh
}

\begin{abstract}
We present a multi-stage Roe-type numerical scheme for a model of two-phase flows arisen from the modeling of deflagration-todetonation transition in granular materials. The first stage in the construction of the scheme computes the volume fraction at every time step. The second stage deals with the nonconservative terms in the governing equations which produces states on both side of the contact wave at each node. In the third stage, a Roe matrix for the two-phase is used to apply on the states obtained from the second stage. This scheme is shown to capture stationary waves and preserves the positivity of the volume fractions. Finally, we present numerical tests which all indicate that the proposed scheme can give very good approximations to the exact solution.
\end{abstract}

\section{Introduction}

Numerical approximations for multi-phase flow models have shown great challenging problems for a quite long time. In this work, we aim to build a Roe-type scheme for the following model of two-phase flows (see [7]),

$$
\begin{aligned}
& \partial_{t}\left(\alpha_{g} \rho_{g}\right)+\partial_{x}\left(\alpha_{g} \rho_{g} u_{g}\right)=0, \\
& \partial_{t}\left(\alpha_{g} \rho_{g} u_{g}\right)+\partial_{x}\left(\alpha_{g}\left(\rho_{g} u_{g}^{2}+p_{g}\right)\right)=p_{g} \partial_{x} \alpha_{g}, \\
& \partial_{t}\left(\alpha_{s} \rho_{s}\right)+\partial_{x}\left(\alpha_{s} \rho_{s} u_{s}\right)=0, \\
& \partial_{t}\left(\alpha_{s} \rho_{s} u_{s}\right)+\partial_{x}\left(\alpha_{s}\left(\rho_{s} u_{s}^{2}+p_{s}\right)\right)=-p_{g} \partial_{x} \alpha_{g}, \\
& \partial_{t} \rho_{s}+\partial_{x}\left(\rho_{s} u_{s}\right)=0, \quad x \in \mathbb{R}, t>0 .
\end{aligned}
$$

The model (1.1) is obtained from the one in [7], which treats the modeling of deflagration-to-detonation transition in granular materials. Here, we assume that the flows are nonreactive and isentropic so that only the equations of conservation of mass and momentum are involved, and the exchange of mass between the two phases are ignored. The first and the third equation of (1.1) expresses the conservation of mass in each phase; the second and the four equation of (1.1) expresses the balance of momentum in each phase; the last

Received April 23, 2013; Revised October 6, 2013.

2010 Mathematics Subject Classification. 35L65, 65M06, 76T10.

Key words and phrases. two-phase flow, balance law, nonconservative, source term, numerical approximation, well-balanced scheme, Roe-type scheme, shock wave, rarefaction wave, contact discontinuity. 
equation of (1.1) is the so-called compaction dynamics equation. See also [4], where an alternative form of the compaction dynamics equation is given. Throughout, the subscripts $g$ and $s$ are used to indicate the quantities in the gas phase and in the solid phase, respectively. Then, $\alpha_{k}, \rho_{k}, u_{k}, p_{k}, k=g, s$, stand for the volume fraction, density, velocity, and pressure in the $k$-phase, $k=g, s$, respectively. The volume fractions are constraint by the relation

$$
\alpha_{s}+\alpha_{g}=1
$$

Roe matrices are widely used in approximating solutions of hyperbolic systems of conservation laws, and in particular, gas dynamics equations. The Roe schemes have been shown to have good accuracy, and could well capture shock waves. However, the model (1.1) has the form of nonconservative systems of balance laws (see [9]). Nonconservative terms in such a system often cause lots of inconvenience for standard numerical approximations. Fortunately, the system (1.1) is hyperbolic, and so this motivates us to develop the Roe scheme technique for the nonconservative system (1.1).

In this paper, we perform several stages in building a Roe-type scheme for (1.1). First, we observe that the nonconservative terms caused by the change of the volume fractions only. By a decomposition approach, we show that the velocity in the solid phase remains constant across contact discontinuities, and that the volume fractions change only across contact discontinuities. An alternative form of the compaction dynamics equation is used where the nonconservative term $u_{s} \partial_{x} \alpha_{g}$ can be numerically treated by an upwind scheme technique. This stage gives us the volume fractions at each time step. Second, we deal with the nonconservative terms in the governing equations of (1.1) by using the contact waves at each node. This stage gives us the states on both sides of the contact wave at each node. Third, we set a Roe matrix for the decoupling system where the volume fractions are removed from the governing equations of (1.1). Then, we substitute the states $U_{j \pm 1}^{n}$ by the states $U_{j \pm 1, \mp}^{n}$ on the other side of the contacts at $x_{j \pm 1 / 2}$ in that Roe matrix of the decoupling system. This completes the three-stage construction of the indirect Roe-type scheme for (1.1). We then prove that the composite scheme is well-balanced and preserves the positivity of the volume fractions. Finally, we provide numerical tests in which we compared the approximate solutions by this Roe-type scheme with the exact solution of the Riemann problem. All tests show very good approximations to the exact solutions.

There have been many works in the literature on numerical approximations of nonconservative systems of balanced laws. The reader is referred to $[1,11$, $16,20,24,25]$ for numerical schemes for multi-phase multi-pressure models. In $[33,34]$, well-balanced numerical schemes for two-phase flow models were built by using stationary waves to track the source terms. Well-balanced numerical schemes for one-pressure models of two-phase flows were constructed in $[30,31]$. Besides, numerical well-balanced schemes for a single conservation law with a source term are presented in $[3,5,6,13,14]$. In $[18,19]$ a well-balanced scheme 
for the model of fluid flows in a nozzle with variable cross-section was were built and studied. Numerical treatments of source terms for shallow water equations were constructed in $[3,8,15,23,32]$. The Riemann problem for models of a fluid in a nozzle with discontinuous cross-section was solved in $[21,28]$. The Riemann problem for shallow water equations with discontinuous topography were solved in $[22,23]$. The Riemann problem for a general system in nonconservative form was studied by [12]. The Riemann problem for twophase flow models were considered in $[2,26,27]$. In our recent work [27], the Riemann problem is solved by using a phase decomposition approach. Twofluid models of two-phase flows were studied in $[17,29]$. See also the references therein.

The organization of this paper is as follows. Section 2 is devoted to basic concepts of the system (1.1) such as characteristic fields, shock waves, rarefaction waves, and the Roe scheme for the case where the volume fractions are constant. In Section 3 we investigate contact waves: the jump relations that determine contact discontinuities, admissibility condition, and the computing algorithm. Section 4 deal with the construction of a Roe-type scheme for the system (1.1). In Section 5 we present numerical tests where the approximate solutions are compared with the exact solutions of the Riemann problem for (1.1). Finally, Section 6 is devoted to concluding remarks.

\section{Preliminaries}

\subsection{Nonstrict hyperbolicity}

Let us assume for simplicity that the flow is isentropic and ideal so that the equations of state are given by

$$
p_{k}=\kappa_{k} \rho_{k}^{\gamma_{k}}, \quad \kappa_{k}>0, \gamma_{k}>1, \quad k=s, g .
$$

The system (1.1) may be re-written as a system of balance laws in nonconservative form as

$$
U_{t}+A(U) U_{x}=0
$$

where

$$
U=\left(\begin{array}{c}
\rho_{g} \\
u_{g} \\
\rho_{s} \\
u_{s} \\
\alpha_{g}
\end{array}\right), \quad A(U)=\left(\begin{array}{ccccc}
u_{g} & \rho_{g} & 0 & 0 & \frac{\rho_{g}\left(u_{g}-u_{s}\right)}{\alpha_{g}} \\
h_{g}^{\prime}\left(\rho_{g}\right) & u_{g} & 0 & 0 & 0 \\
0 & 0 & u_{s} & \rho_{s} & 0 \\
0 & 0 & h_{s}^{\prime}\left(\rho_{s}\right) & u_{s} & \frac{p_{g}-p_{s}}{\alpha_{s} \rho_{s}} \\
0 & 0 & 0 & 0 & u_{s}
\end{array}\right)
$$

and

$$
h_{k}\left(\rho_{k}\right)=\frac{\kappa_{k} \gamma_{k}}{\gamma_{k}-1} \rho_{k}^{\gamma_{k}-1}, \quad k=s, g
$$


Notice that $h_{k}$ satisfies

$$
h_{k}^{\prime}(\rho)=\frac{p_{k}^{\prime}(\rho)}{\rho}, \quad k=s, g .
$$

The matrix $A(U)$ has five eigenvalues

$$
\begin{array}{lll}
\lambda_{1}(U)=u_{g}-\sqrt{p_{g}^{\prime}}, & \lambda_{2}(U)=u_{g}+\sqrt{p_{g}^{\prime}}, & \\
\lambda_{3}(U)=u_{s}-\sqrt{p_{s}^{\prime}}, & \lambda_{4}(U)=u_{s}+\sqrt{p_{s}^{\prime}}, & \lambda_{5}(U)=u_{s} .
\end{array}
$$

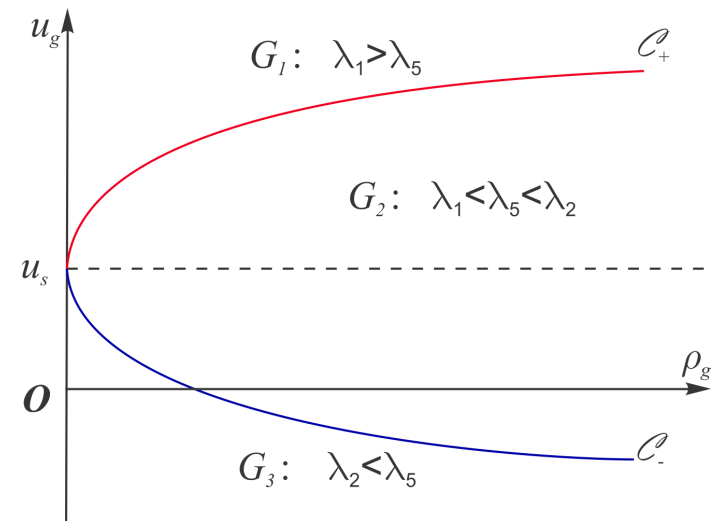

Figure 1. The phase domain in the plane $u_{s} \equiv$ constant

It is interesting that the eigenvalues $\lambda_{5}$ may coincide with either $\lambda_{1}$ or $\lambda_{2}$ on a certain hyper-surface of the phase domain, called the resonant surface. Due to the change of order of these eigenvalues, we set

$$
\begin{aligned}
& G_{1}:=\left\{U \mid \lambda_{1}(U)>\lambda_{5}(U)\right\}, \\
& G_{2}:=\left\{U \mid \lambda_{1}(U)<\lambda_{5}(U)<\lambda_{2}(U)\right\}, \\
& G_{3}:=\left\{U \mid \lambda_{2}(U)<\lambda_{5}(U)\right\}, \\
& \mathcal{C}_{+}:=\left\{U \mid \lambda_{1}(U)=\lambda_{5}(U)\right\}, \\
& \mathcal{C}_{-}:=\left\{U \mid \lambda_{2}(U)=\lambda_{5}(U)\right\} .
\end{aligned}
$$

See Figure (1). The system thus fails to be strictly hyperbolic on the resonant surface

$$
\mathcal{C}:=\mathcal{C}_{+} \cup \mathcal{C}_{-} .
$$

In each region $G_{i}, i=1,2,3$, the system is strictly hyperbolic.

\subsection{Jump relations and the change of volume fraction}

In this subsection, we will show that among elementary waves (shocks, rarefaction waves, and contact discontinuities), the volume fractions change only across the contact discontinuities associated with the fifth characteristic field. 
This suggests us to use the contact waves to track the nonconservative terms on the right-hand side of (1.1).

First, consider rarefaction waves of the system (1.1). These waves are the continuous piecewise-smooth self-similar solutions of (1.1) associated with nonlinear characteristic fields, which have the form

$$
U(x, t)=V(\xi), \quad \xi=\frac{x}{t}, \quad t>0, x \in \mathbb{R} .
$$

Any rarefaction wave satisfies the following initial-value problem for ordinary differential equations

$$
\begin{aligned}
& \frac{d V(\xi)}{d \xi}=r_{i}(V(\xi)), \quad \xi \geq \lambda_{i}\left(U_{0}\right), \quad i=1,2,3,4, \\
& V\left(\lambda_{i}\left(U_{0}\right)\right)=U_{0} .
\end{aligned}
$$

Thus, the integral curve of the first characteristic field is given by

$$
\begin{aligned}
& \frac{d \rho_{g}(\xi)}{d \xi}=\frac{-2 \sqrt{p_{g}^{\prime}\left(\rho_{g}\right)}}{p_{g}^{\prime \prime}\left(\rho_{g}\right) \rho_{g}+2 p_{g}^{\prime}\left(\rho_{g}\right)} \rho_{g}(\xi), \\
& \frac{d u_{g}(\xi)}{d \xi}=\frac{2 \sqrt{p_{g}^{\prime}\left(\rho_{g}\right)}}{p_{g}^{\prime \prime}\left(\rho_{g}\right) \rho_{g}+2 p_{g}^{\prime}\left(\rho_{g}\right)} \sqrt{p_{g}^{\prime}(\xi)}, \\
& \frac{d \rho_{s}(\xi)}{d \xi}=\frac{d u_{s}(\xi)}{d \xi}=\frac{d \alpha_{g}(\xi)}{d \xi}=0 .
\end{aligned}
$$

The last equation means that $\alpha_{g}$ and $\alpha_{s}=1-\alpha_{g}$ are constant through 1 rarefaction waves. Similar argument shows that $\alpha_{g}$ and $\alpha_{s}$ are constant across any rarefaction wave.

Next, consider a discontinuity of (1.1) of the form

$$
U(x, t)= \begin{cases}U_{-} & \text {for } \quad x<\sigma t, \\ U_{+} & \text {for } \quad x>\sigma t,\end{cases}
$$

where $U_{ \pm}$are the left-hand and right-hand states, and $\sigma$ is the speed of discontinuity propagation. This discontinuity in particular satisfies the usual jump relations for the two conservative equations of mass conservation and compaction dynamics:

$$
\begin{aligned}
& -\sigma\left[\alpha_{s} \rho_{s}\right]+\left[\alpha_{s} \rho_{s} u_{s}\right]=0, \\
& -\sigma\left[\rho_{s}\right]+\left[\rho_{s} u_{s}\right]=0,
\end{aligned}
$$

where $[A]=A_{+}-A_{-}$, and $A_{ \pm}$denote the values on the right and left of the jump on the quantity $A$. The equation (2.10) can be rewritten as

or

$$
\begin{aligned}
& {\left[\alpha_{s} \rho_{s}\left(u_{s}-\sigma\right)\right]=0,} \\
& {\left[\rho_{s}\left(u_{s}-\sigma\right)\right]=0,}
\end{aligned}
$$

$$
\begin{aligned}
& \rho_{s}\left(u_{s}-\sigma\right)=M=\text { constant } \\
& M\left[\alpha_{s}\right]=0 .
\end{aligned}
$$


The second equation of (2.11) implies that either $M=0$ or $\left[\alpha_{s}\right]=0$. Since $\rho_{s}>0$, one obtains the following conclusion: across any discontinuity (2.9) of

$$
\text { either }\left[\alpha_{s}\right]=0, \quad \text { or } u_{s}=\sigma=\text { constant. }
$$

It is derived from (2.12) that if $\left[\alpha_{s}\right]=0$, then the volume fractions remain constant across the discontinuity. The governing equations of the system (1.1) is therefore reduced to the decoupling system of two independent sets of isentropic gas dynamics equations in both phases

$$
\partial_{t} V+\partial_{x} F(V)=0,
$$

where

$$
V=\left(\begin{array}{c}
\rho_{g} \\
\rho_{g} u_{g} \\
\rho_{s} \\
\rho_{s} u_{s}
\end{array}\right), \quad F(V)=\left(\begin{array}{c}
\rho_{g} u_{g} \\
\rho_{g} u_{g}^{2}+p_{g} \\
\rho_{s} u_{s} \\
\rho_{s} u_{s}^{2}+p_{s}
\end{array}\right)
$$

From (2.13), one obtains four Hugoniot curves $\mathcal{H}_{i}\left(U_{0}\right), i=1,2,3,4$ and also four corresponding shock curves $\mathcal{S}_{i}\left(U_{0}\right), i=1,2,3,4$. Thus, the volume fractions also remain constant across the $i$-shock waves $i=1,2,3,4$. The remaining Hugoniot curve for the $5 \mathrm{fth}$ characteristic field corresponds to the case $\left[\alpha_{s}\right] \neq 0$ and $u_{s} \equiv$ constant.

Let us fix one state $U_{0}$ and look for any state $U$ that can be connected with $U_{0}$ by a contact discontinuity. As shown in [27], the state $U$ satisfies the equations

$$
\begin{aligned}
& u_{s}=u_{s 0}=\sigma, \\
& \alpha_{g} \rho_{g}\left(u_{g}-u_{s}\right)=\alpha_{g 0} \rho_{g 0}\left(u_{g 0}-u_{s}\right):=m, \\
& \left(u_{g}-u_{s}\right)^{2}+2 h_{g}=\left(u_{g 0}-u_{s 0}\right)^{2}+2 h_{g 0},
\end{aligned}
$$

and

$$
p_{s}=\frac{\alpha_{s 0} p_{s 0}-\left[m u_{g}+\alpha_{g} p_{g}\right]}{\alpha_{s}} .
$$

Observe that the results similar to (2.15) and (2.16) were obtained in [2, 10, 34] for an alternative model of two-phase flows, where the compaction dynamics is given under another form.

The quantities in the gas phase can be found using (2.15). Then, the solid pressure is given by $(2.16)$, and therefore the solid density can be calculated by using the equation of state (2.1).

\section{Admissible contact waves and their computing algorithm}

\subsection{Admissibility criterion}

For simplicity, in the rest of this section we will drop the subindex " $g$ " for the quantities in the gas phase. It follows from (2.15) that the gas density is a 
root of the nonlinear algebraic equation

$$
\begin{aligned}
& \Phi\left(U_{0}, \rho, \alpha\right) \\
(3.1):= & \operatorname{sgn}\left(u_{0}-u_{s}\right)\left(\left(u_{0}-u_{s}\right)^{2}-\mu\left(\rho^{\gamma-1}-\rho_{0}^{\gamma-1}\right)\right)^{1 / 2} \rho-\frac{\alpha_{0}\left(u_{0}-u_{s}\right) \rho_{0}}{\alpha} \\
= & 0,
\end{aligned}
$$

where

$$
\mu=\frac{2 \kappa \gamma}{\gamma-1}
$$

The domain of the function $\Phi\left(U_{0}, \rho, \alpha\right)$ is given by the constraint

$$
\left(u_{0}-u_{s}\right)^{2} \geq \mu\left(\rho^{\gamma-1}-\rho_{0}^{\gamma-1}\right),
$$

or

$$
\rho \leq \bar{\rho}\left(U_{0}\right):=\left(\frac{1}{\mu}\left(u_{0}-u_{s}\right)^{2}+\rho_{0}^{\gamma-1}\right)^{\frac{1}{\gamma-1}} .
$$

Consider first the case $u_{0}-u_{s}>0$. A straightforward calculation shows that

$$
\frac{\partial \Phi\left(U_{0}, \rho ; \alpha\right)}{\partial \rho}=\frac{\left(u_{0}-u_{s}\right)^{2}-\mu\left(\rho^{\gamma-1}-\rho_{0}^{\gamma-1}\right)-\kappa \gamma \rho^{\gamma-1}}{\left(\left(u_{0}-u_{s}\right)^{2}-\mu\left(\rho^{\gamma-1}-\rho_{0}^{\gamma-1}\right)\right)^{1 / 2}}
$$

which yields

where

$$
\begin{array}{ll}
\frac{\partial \Phi\left(U_{0}, \rho ; \alpha\right)}{\partial \rho}>0, & \rho<\rho_{\max }\left(\rho_{0}, u_{0}\right) \\
\frac{\partial \Phi\left(U_{0}, \rho ; \alpha\right)}{\partial \rho}<0, & \rho>\rho_{\max }\left(\rho_{0}, u_{0}\right)
\end{array}
$$

$$
\rho_{\max }\left(\rho_{0}, u_{0}\right):=\left(\frac{2}{\mu(\gamma+1)}\left(u_{0}-u_{s}\right)^{2}+\frac{2}{\gamma+1} \rho_{0}^{\gamma-1}\right)^{\frac{1}{\gamma-1}} .
$$

The values of $\Phi$ at the limits are given by

$$
\Phi\left(U_{0}, \rho=0, \alpha\right)=\Phi\left(U_{0}, \rho=\bar{\rho}, \alpha\right)=-\frac{\alpha_{0}\left(u_{0}-u_{s}\right) \rho_{0}}{\alpha}<0 .
$$

Thus, the function $\rho \mapsto \Phi\left(U_{0}, \rho ; \alpha\right)$ admits a root if and only if the maximum value is non-negative:

$$
\Phi\left(U_{0}, \rho=\rho_{\max }, \alpha\right) \geq 0
$$

In other words,

$$
\alpha \geq \alpha_{\min }\left(U_{0}\right):=\frac{\alpha_{0} \rho_{0}\left|u_{0}-u_{s}\right|}{\sqrt{\kappa \gamma} \rho_{\max }^{\frac{\gamma+1}{2}}\left(\rho_{0}, u_{0}\right)} .
$$

Similar argument can be made for $u_{0}-u_{s}<0$. If we replace $U_{0}$ by $U$ in (3.3), we can define a function $\alpha_{\min }\left(\alpha, \rho, u ; u_{s}\right)$ for each fixed $u_{s}$ by

$$
\alpha_{\min }\left(\alpha, \rho, u ; u_{s}\right):=\frac{\alpha \rho\left|u-u_{s}\right|}{\sqrt{\kappa \gamma} \rho_{\max }^{\frac{\gamma+1}{2}}(\rho, u)} .
$$



(3.1).

The following results characterize the properties of roots of the equation

Lemma 3.1. (i) The function $\Phi\left(U_{0}, \rho, \alpha\right)$ in (3.1) admits a zero if and only if $a \geq \alpha_{\min }\left(U_{0}\right)$. In this case, $\Phi\left(U_{0}, \rho, \alpha\right)$ admits two distinct zeros, denoted by $\rho=\varphi_{1}\left(U_{0}, \alpha\right), \rho=\varphi_{2}\left(U_{0}, \alpha\right)$ such that

$$
\varphi_{1}\left(U_{0}, \alpha\right) \leq \rho_{\max }\left(U_{0}\right) \leq \varphi_{2}\left(U_{0}, \alpha\right),
$$

where the equality holds only if $\alpha=\alpha_{\min }\left(U_{0}\right)$.

(ii) Let $\rho_{\max }\left(\rho_{0}, u_{0}\right)$ be defined as (3.2). Then, it holds that

$$
\begin{array}{ll}
\rho_{\max }\left(\rho_{0}, u_{0}\right)<\rho_{0}, & \left(\rho_{0}, u_{0}\right) \in G_{2}, \\
\rho_{\max }\left(\rho_{0}, u_{0}\right)>\rho_{0}, & \left(\rho_{0}, u_{0}\right) \in G_{1} \cup G_{3}, \\
\rho_{\max }\left(\rho_{0}, u_{0}\right)=\rho_{0}, & \left(\rho_{0}, u_{0}\right) \in \mathcal{C} .
\end{array}
$$

(iii) The state $\left(\varphi_{1}\left(U_{0}, \alpha\right), u=u_{s}+m / \alpha \rho\right) \in G_{1}$ if $u_{0}-u_{s}<0$, and the state $\left(\varphi_{1}\left(U_{0}, \alpha\right), u=u_{s}+m / \alpha \rho\right) \in G_{3}$ if $u_{0}-u_{s}>0$; the state $\left(\varphi_{2}\left(U_{0}, \alpha\right), u=\right.$ $\left.u_{s}+m / \alpha \rho\right) \in G_{2}$. Moreover,

$$
\left(\rho_{\max }\left(\rho_{0}, u_{0}\right), u_{0}\right) \in \mathcal{C} .
$$

In addition, we have

- If $\alpha>\alpha_{0}$, then

$$
\varphi_{1}\left(U_{0}, \alpha\right)<\rho_{0}<\varphi_{2}\left(U_{0}, \alpha\right)
$$

- If $\alpha<\alpha_{0}$, then

$$
\begin{array}{lll}
\rho_{0}<\varphi_{1}\left(U_{0}, \alpha\right) & \text { for } & \left(\rho_{0}, u_{0}\right) \in G_{1} \cup G_{3}, \\
\rho_{0}>\varphi_{2}\left(U_{0}, \alpha\right) & \text { for } & \left(\rho_{0}, u_{0}\right) \in G_{2} .
\end{array}
$$

(iv) For each fixed $u_{s}$, the quantity $\alpha_{\min }\left(\alpha, \rho, u ; u_{s}\right)$ defined by (3.4) attains its strict maximum value $\alpha$ on the surface $\mathcal{C}$. Moreover, we can express

$$
\alpha_{\min }\left(\alpha, \rho, u ; u_{s}\right)=\eta(\theta), \quad \theta:=\left|u-u_{s}\right| / \rho^{(\gamma-1) / 2},
$$

where the function $\eta$ is strictly increasing for $0 \leq \theta \leq \sqrt{\kappa \gamma}$, and strictly decreasing for $\theta>\sqrt{\kappa \gamma}$, and that

$$
\alpha_{\min }\left(\alpha, \rho, u=0 ; u_{s}\right)=0, \quad \lim _{\theta \rightarrow \infty} \eta(\theta)=0 .
$$

This means that $\alpha_{\min }\left(\alpha, \rho, u ; u_{s}\right)$ is strictly decreasing as the point $(\rho, u)$ is moving far away from the surface $\mathcal{C}$.

Proof. The proof of (i)-(iii) are omitted, since it is similar to the one in [21]. We now prove (iv). It holds that

$$
\alpha_{\min }\left(\alpha, \rho, u ; u_{s}\right)=\frac{\alpha \rho\left|u-u_{s}\right|}{\sqrt{\kappa \gamma}\left\{\frac{\kappa \gamma(\gamma+1)}{\gamma-1}\left(\left(u-u_{s}\right)^{2}+\frac{2 \kappa \gamma}{\gamma-1} \rho^{\gamma-1}\right)\right\}^{\frac{\gamma+1}{2(\gamma-1)}}} .
$$


Substituting $\left|u-u_{s}\right|=\theta \rho^{(\gamma-1) / 2}$ into (3.5) gives us

$$
\alpha_{\min }\left(\alpha, \rho, u ; u_{s}\right)=\frac{\alpha \rho^{(\gamma+1) / 2} \theta}{\sqrt{\kappa \gamma}\left\{\frac{\kappa \gamma(\gamma+1)}{\gamma-1}\left(\theta^{2}+\frac{2 \kappa \gamma}{\gamma-1}\right) \rho^{\gamma-1}\right\}^{\frac{\gamma+1}{2(\gamma-1)}}} .
$$

Simplifying the last expression yields

$$
\eta(\theta):=\alpha_{\min }\left(\alpha, \rho, u ; u_{s}\right)=\frac{\alpha \theta}{m\left(\theta^{2}+\frac{2 \kappa \gamma}{\gamma-1}\right)^{\frac{\gamma+1}{2(\gamma-1)}}},
$$

where

$$
m:=\frac{1}{\sqrt{\kappa \gamma}\left(\frac{\kappa \gamma(\gamma+1)}{\gamma-1}\right)^{\frac{\gamma+1}{2(\gamma-1)}}}
$$

A straightforward calculation gives

$$
\frac{d \eta}{d \theta}=\frac{2 \alpha}{(\gamma-1) m\left(\theta^{2}+\frac{2 \kappa \gamma}{\gamma-1}\right)^{\frac{\gamma+1}{2(\gamma-1)}+1}}\left(\kappa \gamma-\theta^{2}\right) .
$$

This implies that

$$
\begin{aligned}
& \frac{d \eta}{d \theta}>0, \quad 0<\theta<\sqrt{\kappa \gamma}, \\
& \frac{d \eta}{d \theta}<0, \quad \theta>\sqrt{\kappa \gamma} \\
& \frac{d \eta}{d \theta}=0, \quad \theta=\sqrt{\kappa \gamma},
\end{aligned}
$$

which means that the function $\eta$ is strictly increasing for $0 \leq \theta<\sqrt{\kappa \gamma}$, strictly decreasing for $\theta \geq \sqrt{\kappa \gamma}$, and attains its strict maximum at $\theta=\sqrt{\kappa \gamma}$.

It follows from (3.6) that

$$
\alpha_{\min }\left(\alpha, \rho, u=0 ; u_{s}\right)=0, \quad \lim _{\theta \rightarrow \infty} \eta(\theta)=0 .
$$

The proof of Lemma 3.1 is complete.

In view of Lemma 3.1, given a state $U_{0}$, there may be two states $U$ on the other side of a contact discontinuity from $U_{0}$. Thus, it is natural to impose an admissibility condition to select the meaningful solution. Following $[21,28,27]$, we impose the following admissibility criterion for contact discontinuities.

(MC) Any contact wave does not cross the resonant surface $\mathcal{C}$.

Thus, Lemma 3.1 and the (MC) criterion yield the following result that determines the admissible root of (3.1) and therefore the admissible contact satisfying the (MC) criterion.

Lemma 3.2. (i) If $U_{0} \in G_{1} \cup G_{3}$, then only the zero $\rho=\varphi_{1}\left(U_{0}, \alpha\right)$ is selected. 
(ii) If $U_{0} \in G_{2}$, then only the zero $\rho=\varphi_{2}\left(U_{0}, \alpha\right)$ is selected.

\subsection{Computing algorithm}

The function $\rho \mapsto \Phi\left(U_{0}, \alpha ; \rho\right)$ in (3.1) helps us to determine the admissible contact wave. However, since this function is not convex, Newton's method for the nonlinear equation (3.1) may not converge. In this subsection we will present a numerical method to compute the admissible root among the two roots $\varphi_{i}\left(U_{0}, \alpha\right), i=1,2$ defined in Lemma 3.1. Our purpose is to derive from (2.15) a nonlinear equation

$$
f(\rho)=0,
$$

where $f$ is a strictly convex function, and determine a convenient starting point for which Newton's method converge to the admissible root. To deal with this, we move the last term of (3.1) to the right-hand side, take the square both sides, then multiply both sides of the resulted equation by $1 / \rho$, then move the right-hand side term to the left-hand side, and finally re-arrange terms to get

$\Psi\left(U_{0}, \alpha ; \rho\right):=\mu \rho^{\gamma}-\left(\left(u_{0}-u_{s}\right)^{2}+\mu \rho_{0}^{\gamma-1}\right) \rho+\left(\frac{\alpha_{0}\left(u_{0}-u_{s}\right) \rho_{0}}{\alpha}\right)^{2} \frac{1}{\rho}=0, \quad \rho>0$,

where

$$
\mu=\frac{2 \kappa \gamma}{\gamma-1}
$$

The function $\Psi$ in (3.7) is strictly convex. One can easily check this by taking a straightforward calculation that gives

$$
\begin{aligned}
& \frac{d \Psi\left(U_{0}, \alpha ; \rho\right)}{d \rho}=\mu \gamma \rho^{\gamma-1}-\left(\left(u_{0}-u_{s}\right)^{2}+\mu \rho_{0}^{\gamma-1}\right)-\left(\frac{\alpha_{0}\left(u_{0}-u_{s}\right) \rho_{0}}{\alpha}\right)^{2} \frac{1}{\rho^{2}}, \\
& \frac{d^{2} \Psi\left(U_{0}, a ; \rho\right)}{d \rho^{2}}=\mu \gamma(\gamma-1) \rho^{\gamma-2}+2\left(\frac{\alpha_{0}\left(u_{0}-u_{s}\right) \rho_{0}}{\alpha}\right)^{2} \frac{1}{\rho^{3}}>0, \quad \rho>0 .
\end{aligned}
$$

Therefore, Newton's method could be suitable for the nonlinear equation (3.7). However, we still have to make sure that the starting point is selected such that the derivative of $d \Psi / d \rho$ does not vanish during the computation. To deal with this, we first observe that

$$
\Psi\left(U_{0}, \alpha ; \rho\right) \Phi\left(U_{0}, \alpha ; \rho\right)<0, \quad 0<\rho \neq \varphi_{i}\left(U_{0}, \alpha\right), i=1,2 .
$$

As seen in the previous subsection, we have

$$
\Psi\left(U_{0}, \alpha ; \rho_{0}\right)<0, \quad \varphi_{1}\left(U_{0}, \alpha\right)<\rho_{0}<\varphi_{2}\left(U_{0}, \alpha\right) .
$$

Thus, to compute the root $\varphi_{1}\left(U_{0}, \alpha\right)$, we can use Newton's method to (3.7) with any starting point less than $\varphi_{1}\left(U_{0}, \alpha\right)$. Precisely, it is derived from (3.8) that

$$
\Psi\left(U_{0}, \alpha ; \rho\right)>-\left(\left(u_{0}-u_{s}\right)^{2}+\mu \rho_{0}^{\gamma-1}\right) \rho+\left(\frac{\alpha_{0}\left(u_{0}-u_{s}\right) \rho_{0}}{\alpha}\right)^{2} \frac{1}{\rho} \geq 0
$$


for

$$
\rho \leq \rho_{*}:=\frac{\alpha_{0}\left(u_{0}-u_{s}\right) \rho_{0}}{\alpha \sqrt{\left(u_{0}-u_{s}\right)^{2}+\mu \rho_{0}^{\gamma-1}}}<\varphi_{1}\left(U_{0}, \alpha\right) .
$$

Since

$$
\Psi\left(U_{0}, \alpha ; \rho\right)>0, \frac{d^{2} \Psi\left(U_{0}, \alpha ; \rho\right)}{d \rho^{2}}>0
$$

for $0<\rho<\varphi_{1}\left(U_{0}, \alpha\right)$ and $\rho_{*}<\varphi_{1}\left(U_{0}, \alpha\right)$, Newton's method starting at $\rho_{*}$ will generate a strictly increasing sequence that converges to $\varphi_{1}\left(U_{0}, \alpha\right)$.

Similarly, to compute the root $\varphi_{2}\left(U_{0}, \alpha\right)$, we can use Newton's method with any starting point larger than $\varphi_{2}\left(U_{0}, \alpha\right)$. From (3.8) one has

$$
\Psi\left(U_{0}, \alpha ; \rho\right)>\mu \rho^{\gamma}-\left(\left(u_{0}-u_{s}\right)^{2}+\mu \rho_{0}^{\gamma-1}\right) \rho \geq 0
$$

for

$$
\begin{aligned}
\rho \geq \rho^{*} & :=\left(\frac{\left(u_{0}-u_{s}\right)^{2}}{\mu}+\rho_{0}^{\gamma-1}\right)^{1 /(\gamma-1)}=\left(\frac{\gamma+1}{2}\right)^{1 /(\gamma-1)} \rho_{\max }\left(U_{0}, \alpha\right) \\
& >\varphi_{2}\left(U_{0}, \alpha\right)
\end{aligned}
$$

Since

$$
\Psi\left(U_{0}, \alpha ; \rho\right)>0, \frac{d^{2} \Psi\left(U_{0}, \alpha ; \rho\right)}{d \rho^{2}}>0
$$

for $\rho>\varphi_{2}\left(U_{0}, \alpha\right)$ and $\rho^{*}>\varphi_{2}\left(U_{0}, \alpha\right)$, Newton's method starting at $\rho^{*}$ will generate a strictly decreasing sequence that converges to $\varphi_{2}\left(U_{0}, \alpha\right)$.

\section{Algorithm for computing the admissible root}

Based on the above argument, we can determine an algorithm for computing the admissible root of (3.7) and therefore the admissible contact satisfying (2.15) as follows.

Lemma 3.3 (Computing admissible contact). Let $U_{0}=\left(\rho_{g 0}, u_{g 0}, \rho_{s 0}, u_{s 0}, \alpha_{g 0}\right)^{t}$ be an arbitrarily fixed state. The admissible state $U=\left(\rho_{g}, u_{g}, \rho_{s}, u_{s}, \alpha_{g}\right)^{t}$ that can be connected with $U_{0}$ by a contact discontinuity can be determined as follows. First, the gas density can be found using Newton's method for (3.7) in the following way:

- If $U_{0} \in G_{1} \cup G_{3}$, start Newton's method at $\rho=\rho_{*}$ given by (3.9);

- Otherwise, start Newton's method at $\rho=\rho^{*}$ given by (3.10).

Then, the other quantities of the admissible contact are computed from the relations (2.15) and (2.16). If $U_{0}=U_{-}$is chosen to be the left-hand state of the contact, then the corresponding right-hand state is $U_{+}=U$. If $U_{0}=U_{+}$ is the right-hand state of the contact, then the corresponding left-hand state is 
$U_{-}=U$. The states $U_{ \pm}$will determine the values

$$
V_{ \pm}:=\left(\begin{array}{c}
\rho_{g, \pm} \\
\rho_{g, \pm} u_{g, \pm} \\
\rho_{s, \pm} \\
\rho_{s, \pm} u_{s, \pm}
\end{array}\right)
$$

on both sides of the contact discontinuity.

The points $V_{ \pm}$in (3.11) will be used in the construction of the Roe-type scheme in the next section.

\section{A well-balanced Roe-type scheme}

Denote by $\Delta t$ the time step, and by $\Delta x$ the spacial mesh size, which satisfy the following C.F.L. stability condition

$$
\lambda \max _{U}\left\{\left|u_{g}\right|+\sqrt{p_{g}^{\prime}\left(\rho_{g}\right)},\left|u_{s}\right|+\sqrt{p_{s}^{\prime}\left(\rho_{s}\right)}\right\}<1, \quad \lambda:=\frac{\Delta t}{\Delta x} .
$$

\subsection{Roe's scheme for the decoupling system}

A Roe matrix for the decoupling system (2.13) is given by

$$
A\left(U_{L}, U_{R}\right)=\left(\begin{array}{cccc}
0 & 1 & 0 & 0 \\
-\bar{u}_{g}{ }^{2}+\bar{p}_{g} & 2 \bar{u}_{g} & 0 & 0 \\
0 & 0 & 0 & 1 \\
0 & 0 & -\bar{u}_{s}^{2}+\bar{p}_{s} & 2 \bar{u}_{s}
\end{array}\right),
$$

where

$$
\bar{u}=\frac{\sqrt{\rho_{L}} u_{L}+\sqrt{\rho_{R}} u_{R}}{\sqrt{\rho_{L}}+\sqrt{\rho_{R}}}, \quad \bar{p}= \begin{cases}\frac{p_{R}-p_{L}}{\rho_{R}-\rho_{L}}, & \text { if } \quad \rho_{L} \neq \rho_{R}, \\ p^{\prime}\left(\rho_{L}\right), & \text { if } \rho_{L}=\rho_{R},\end{cases}
$$

and the notations hold for the corresponding quantities in both phases.

The Roe matrix (4.2) has four eigenvalues

$$
\begin{array}{ll}
\bar{\lambda}_{1}\left(U_{L}, U_{R}\right)=\bar{u}_{g}-\sqrt{\bar{p}_{g}}, & \bar{\lambda}_{2}\left(U_{L}, U_{R}\right)=\bar{u}_{g}+\sqrt{\bar{p}_{g}}, \\
\bar{\lambda}_{3}\left(U_{L}, U_{R}\right)=\bar{u}_{s}-\sqrt{\bar{p}_{s}}, & \bar{\lambda}_{4}\left(U_{L}, U_{R}\right)=\bar{u}_{s}+\sqrt{\bar{p}_{s}} .
\end{array}
$$

The corresponding right-eigenvectors can be chosen as

$$
\bar{r}_{1}\left(U_{L}, U_{R}\right)=\left(\begin{array}{c}
1 \\
\bar{\lambda}_{1}\left(U_{L}, U_{R}\right) \\
0 \\
0
\end{array}\right), \quad \bar{r}_{2}\left(U_{L}, U_{R}\right)=\left(\begin{array}{c}
1 \\
\bar{\lambda}_{2}\left(U_{L}, U_{R}\right) \\
0 \\
0
\end{array}\right),
$$

and

$$
\bar{r}_{3}\left(U_{L}, U_{R}\right)=\left(\begin{array}{c}
0 \\
0 \\
1 \\
\bar{\lambda}_{3}\left(U_{L}, U_{R}\right)
\end{array}\right), \quad \bar{r}_{4}\left(U_{L}, U_{R}\right)=\left(\begin{array}{c}
0 \\
0 \\
1 \bar{\lambda}_{4}\left(U_{L}, U_{R}\right)
\end{array}\right)
$$


The corresponding left-eigenvectors of the Roe matrix can be chosen as

$$
\begin{aligned}
& \bar{l}_{1}\left(U_{L}, U_{R}\right)=\frac{-1}{2 \sqrt{\bar{p}_{g}}}\left(-\bar{\lambda}_{2}\left(U_{L}, U_{R}\right), 1\right), \\
& \bar{l}_{2}\left(U_{L}, U_{R}\right)=\frac{-1}{2 \sqrt{\bar{p}_{g}}}\left(-\bar{\lambda}_{1}\left(U_{L}, U_{R}\right), 1\right), \\
& \bar{l}_{3}\left(U_{L}, U_{R}\right)=\frac{-1}{2{\sqrt{\bar{p}_{s}}}_{(}}\left(-\bar{\lambda}_{4}\left(U_{L}, U_{R}\right), 1\right), \\
& \bar{l}_{4}\left(U_{L}, U_{R}\right)=\frac{-1}{2 \sqrt{\bar{p}_{s}}}\left(-\bar{\lambda}_{3}\left(U_{L}, U_{R}\right), 1\right) .
\end{aligned}
$$

It is easy to check that

$$
\bar{l}_{i}\left(U_{L}, U_{R}\right) \cdot \bar{r}_{j}\left(U_{L}, U_{R}\right)=\delta_{i}^{j}, \quad i, j=1,2,3,4 .
$$

Set the coefficients

$$
\alpha_{i}\left(U_{L}, U_{R}\right)=\bar{l}_{i}\left(U_{L}, U_{R}\right)\left(U_{R}-U_{L}\right), \quad i=1,2,3,4 .
$$

The Roe numerical flux for the system (2.13) is given by

$$
G(V, W)=\frac{1}{2}(F(V)+F(W))-\frac{1}{2} \sum_{i=1}^{4}\left(\left|\bar{\lambda}_{i}\right| \alpha_{i} r_{i}\right)(V, W),
$$

where $V, F(V)$ are given by (2.14). A Roe scheme for the decoupling system for $(2.13)$ can be defined by

$$
V_{j}^{n+1}=V_{j}^{n}-\frac{\Delta t}{\Delta x}\left(G\left(V_{j}^{n}, V_{j+1}^{n}\right)-G\left(V_{j-1}^{n}, V_{j}^{n}\right)\right) .
$$

\subsection{A three-stage construction of a Roe-type scheme}

Let us regroup the system (1.1) into two groups: the equations determining the dynamics of volume fractions

$$
\begin{aligned}
& \partial_{t}\left(\alpha_{s} \rho_{s}\right)+\partial_{x}\left(\alpha_{s} \rho_{s} u_{s}\right)=0 \\
& \partial_{t} \rho_{s}+\partial_{x}\left(\rho_{s} u_{s}\right)=0
\end{aligned}
$$

and the governing equations in both phases

$$
\begin{aligned}
& \partial_{t}\left(\alpha_{g} \rho_{g}\right)+\partial_{x}\left(\alpha_{g} \rho_{g} u_{g}\right)=0 \\
& \partial_{t}\left(\alpha_{g} \rho_{g} u_{g}\right)+\partial_{x}\left(\alpha_{g}\left(\rho_{g} u_{g}^{2}+p_{g}\right)\right)=p_{g} \partial_{x} \alpha_{g}, \\
& \partial_{t}\left(\alpha_{s} \rho_{s}\right)+\partial_{x}\left(\alpha_{s} \rho_{s} u_{s}\right)=0 \\
& \partial_{t}\left(\alpha_{s} \rho_{s} u_{s}\right)+\partial_{x}\left(\alpha_{s}\left(\rho_{s} u_{s}^{2}+p_{s}\right)\right)=-p_{g} \partial_{x} \alpha_{g}
\end{aligned}
$$

In the following we will present a three-stage construction of a Roe-type scheme relying on this regrouping technique. 


\section{First stage: Computing volume fractions}

Multiplying the second equation of (4.4) by $\alpha_{s}$, and then subtracting the result from the first equation of (4.4), one has

$\partial_{t}\left(\alpha_{s} \rho_{s}\right)+\partial_{x}\left(\alpha_{s} \rho_{s} u_{s}\right)-\alpha_{s}\left(\partial_{t}\left(\rho_{s}\right)+\partial_{x}\left(\rho_{s} u_{s}\right)\right)=\rho_{s} \partial_{t}\left(\alpha_{s}\right)+\rho_{s} u_{s} \partial_{x}\left(\alpha_{s}\right)=0$.

Canceling $\rho_{s} \neq 0$ from the last equation, on gets

$$
\partial_{t} \alpha_{s}+u_{s} \partial_{x} \alpha_{s}=0
$$

or, since $\alpha_{g}+\alpha_{s}=1$

$$
\partial_{t} \alpha_{g}+u_{s} \partial_{x} \alpha_{g}=0 .
$$

As seen Section 2 that the volume fraction $\alpha_{g}$ could change only across the $5 \mathrm{fth}$ contact discontinuities while the solid velocity $u_{s}$ still remains constant. Thus, the nonconservative term $u_{s} \partial_{x} \alpha_{g}$ would be in fact regular at least at discrete level. Motivated by this observation, we take an upwind scheme technique for the equation (4.6) to compute the gas volume fraction:

$$
\alpha_{g, j}^{n+1}=\alpha_{g, j}^{n}-\lambda\left(u_{s, j}^{+, n}\left(\alpha_{g, j}^{n}-\alpha_{g, j-1}^{n}\right)+u_{s, j}^{-, n}\left(\alpha_{g, j+1}^{n}-\alpha_{g, j}^{n}\right)\right),
$$

where

$$
u^{+}=\max \{u, 0\}, \quad u^{-}=\min \{u, 0\} .
$$

\section{Second stage: Dealing with nonconservative terms}

First, we observe that when calculating $V_{j}^{n+1}$, a 2-step scheme requires only the states on both sides of the nodes node $x_{j \pm 1 / 2}=(j \pm 1 / 2) \Delta x, t=t^{n}=n \Delta t$, which are merely $V_{j+k}^{n}, k= \pm 1,0$. So, we deal with the nonconservative terms in the governing equations (4.5) across each of this node as follows.

(i) Across the node $x_{j+1 / 2}=(j+1 / 2) \Delta x, t=t^{n}=n \Delta t$, the volume fraction change $\Delta \alpha=\alpha_{j+1}^{n}-\alpha_{j}^{n}$ absorbs the nonconservative terms in (4.5) into a 5-contact discontinuity that jumps from the state $V_{j+1}^{n}$ with the gas volume fraction $\alpha_{j+1}^{n}$ into an admissible state with the gas volume fraction $\alpha_{j}^{n}$ denoted by $V_{j+1,-}^{n}$.

(ii) Across the node $x_{j-1 / 2}=(j-1 / 2) \Delta x, t=t^{n}$, the volume fraction change $\Delta \alpha=\alpha_{j-1}^{n}-\alpha_{j}^{n}$ absorbs the nonconservative terms in (4.5) by a 5-contact discontinuity that jumps from the state $V_{j-1}^{n}$ with the gas volume fraction $\alpha_{j-1}^{n}$ into an admissible state with the gas volume fraction $\alpha_{j}^{n}$, denoted by $V_{j-1,+}^{n}$.

(iii) The computing algorithm for the states $V_{j+1,-}^{n}$ and $V_{j-1,+}^{n}$ is given by Lemma 3.3.

The states $V_{j+1,-}^{n}$ and $V_{j-1,+}^{n}$ are used to absorb the nonconservative terms of the system (1.1). This process, of course, requires a certain computational cost.

Next, it is useful to make the scheme "robust", i.e., it can always work, even if the exact solution may not exist. This is the case if the process of computing 
the states $V_{j \pm 1, \mp}^{n}$ can always be done. So, the volume fraction $\alpha_{g, j}^{n}$ may be substituted by its "relaxed" value defined by

$$
\alpha_{g, j}^{n, \text { Relax }}=\max \left\{\alpha_{g, j}^{n}, \alpha_{\min }\left(\alpha_{g, j \pm 1}^{n}, \rho_{g, j \pm 1}^{n}, u_{g, j \pm 1}^{n} ; u_{s, j \pm 1}^{n}\right)\right\} .
$$

respectively, where $\alpha_{\text {min }}$ is defined by (3.3). As seen by Lemma 3.1, part (iv), it holds that

$$
\alpha_{\min }\left(\alpha_{g, j \pm 1}^{n}, \rho_{g, j \pm 1}^{n}, u_{g, j \pm 1}^{n} ; u_{s, j \pm 1}^{n}\right)<\alpha_{g, j \pm 1}^{n} .
$$

Therefore,

$$
\alpha_{g, j}^{n, \text { Relax }}=\alpha_{g, j}^{n}
$$

and so the scheme is expected to approximate the exact solution, if the change

$$
\left|\alpha_{g, j}^{n}-\alpha_{g, j \pm 1}^{n}\right|
$$

is not so large, at least around the surface $\mathcal{C}$. In particular, if the volume fractions are continuous, a reasonable mesh-size refinement can assure that the change (4.10) is small enough, so that (4.9) holds. Nevertheless, the exact solution may not exist if the sudden change of volume fractions is so large, i.e., the condition (3.3) is violated. In such as a case, due to the "relaxation" (4.8), the scheme still works.

\section{Third stage: Completing a Roe-type scheme}

Since the gas volume fraction at the three states $V_{j}^{n}$ and $V_{j \pm 1, \mp}^{n}$ coincide, it can be considered as a constant (at least) at discrete level and thus can be removed from the second group (4.5). This step transforms (4.5) into the decoupling system (2.12)-(2.13). Then, let $G(U, V)$ be the Roe numerical flux (4.3). Together with (4.7), we define a Roe-type scheme

$$
\begin{aligned}
& V_{j}^{n+1}=V_{j}^{n}-\lambda\left(G\left(V_{j}^{n}, V_{j+1,-}^{n}\right)-G\left(V_{j-1,+}^{n}, V_{j}^{n}\right)\right), \quad \lambda=\frac{\Delta t}{\Delta x}, \\
& \alpha_{g, j}^{n+1}=\alpha_{g, j}^{n}-\lambda\left(u_{s, j}^{+, n}\left(\alpha_{g, j}^{n}-\alpha_{g, j-1}^{n}\right)+u_{s, j}^{-, n}\left(\alpha_{g, j+1}^{n}-\alpha_{g, j}^{n}\right)\right) .
\end{aligned}
$$

The three-stage Roe-type scheme (4.11) possesses nice properties as in the following theorem.

Theorem 4.1. The following conclusions hold

(i) (Positivity of volume fractions) The three-stage Roe-type scheme (4.11) preserves the positivity of the volume fractions. This means that if $\alpha_{k, j}^{0}>0$ for all $j \in \mathbb{Z}$, then $\alpha_{k, j}^{n}>0$ for all $j \in \mathbb{Z}, n=1,2,3, \ldots, k=$ $s, g$.

(ii) (Well-balanced scheme) The three-stage Roe-type scheme (4.11) captures exactly equilibrium states on both sides of any stationary contact discontinuity. 
Proof. The proof of (i) is omitted, since it is similar to the one in Theorem 4.1, [33]. To prove (ii), let us be given a stationary contact discontinuity. Then, $u_{s}=0$. So from (4.7), we deduce that the volume fractions remain unchanged:

$$
\alpha_{s, j}^{n+1}=\alpha_{s, j}^{n}, \quad \forall j, n .
$$

Next, the relations (2.15) hold for this stationary contact discontinuity. Thus, by definition

$$
\begin{array}{ll}
\rho_{g, j+1,-}^{n}=\rho_{g, j}^{n}, & u_{g, j+1,-}^{n}=u_{g, j}^{n}, \\
\rho_{g, j-1,+}^{n}=\rho_{g, j}^{n}, & u_{g, j-1,+}^{n}=u_{g, j}^{n}, \\
\rho_{s, j+1,-}^{n}=\rho_{s, j}^{n}, & u_{s, j+1,-}^{n}=u_{s, j}^{n}, \\
\rho_{s, j-1,+}^{n}=\rho_{s, j}^{n}, & u_{s, j-1,+}^{n}=u_{s, j}^{n},
\end{array}
$$

so that

$$
V_{j+1,-}^{n}=V_{j}^{n}, \quad V_{j-1,+}^{n}=V_{j}^{n} .
$$

This yields

$$
V_{j}^{n+1}=V_{j}^{n},
$$

which establishes (ii). The proof of Theorem 4.1 is complete.

\section{Numerical tests}

This section is devoted to numerical tests. Approximate solutions obtained by the multi-stage Roe-type scheme (4.11) are compared with the exact solutions of the Riemann problem for (1.1). Riemann data are taken in the form

$$
\left(\alpha_{g 0}, p_{g 0}, u_{g 0}, p_{s 0}, u_{s 0}\right)(x)= \begin{cases}\left(\alpha_{g L}, p_{g L}, u_{g L}, p_{s L}, u_{s L}\right) & \text { if } x<0 \\ \left(\alpha_{g R}, p_{g R}, u_{g R}, p_{s R}, u_{s R}\right) & \text { if } x>0\end{cases}
$$

The solution will be computed on the interval $[-1,1]$ of the $x$-space at the time $t=0.1$.

\subsection{Numerical Test 1: Stationary contact discontinuities}

In this test, the equations of state are of the form (2.1), where we take the parameters as

$$
\gamma_{g}=1.4, \quad \gamma_{s}=1.6, \quad \kappa_{g}=0.4, \quad \kappa_{s}=1 .
$$

As seen from Theorem 4.1, the three-stage Roe-type scheme (4.11) is wellbalanced. This test is carried out to verify this property. The approximate solution will be computed at the time $t=0.1$ on the interval $[-1,1]$ of the $x$ space with 1000 mesh points with C.F.L. $=1 / 4$. Consider the Riemann problem for (1.1)-(1.2) with the initial data given in Table 1. 

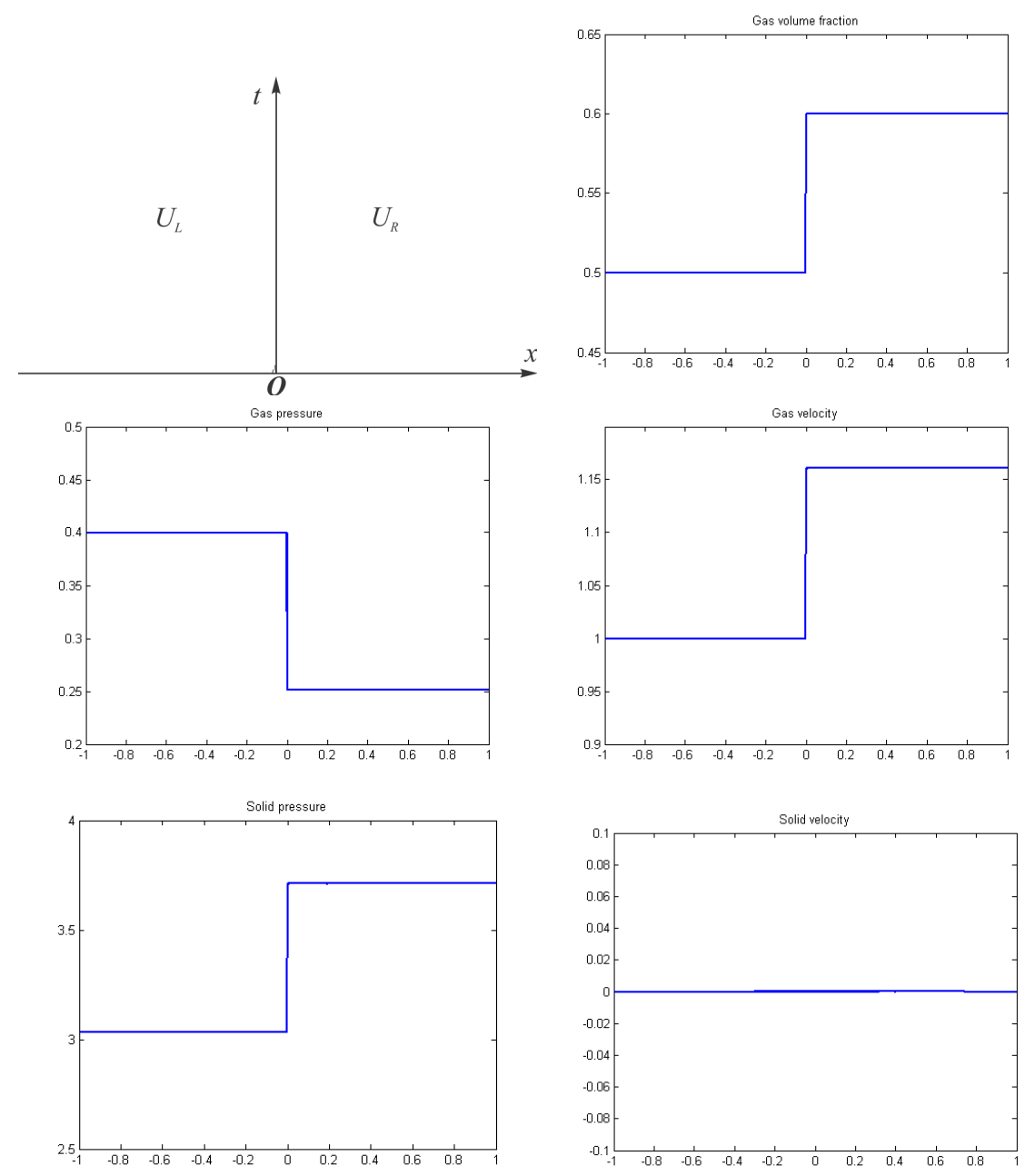

Figure 2. Test 1: The exact stationary contact wave in the $(x, t)$-plane (upper-left corner), and its approximations at the time $t=0.1$ in the interval $x \in[-1,1]$ with 1000 mesh points

\begin{tabular}{|c|c|c|}
\hline \multicolumn{3}{|c|}{ Table 1 } \\
\hline Quantities \States & $U_{L}$ & $U_{R}$ \\
\hline$\alpha_{g}$ & 0.5 & 0.6 \\
$p_{g}$ & 1 & 0.71781502 \\
$u_{g}$ & 1 & 1.1609305 \\
$p_{s}$ & 2 & 2.2694822 \\
$u_{s}$ & 0 & 0 \\
\hline
\end{tabular}


It is not difficult to check that in this case the Riemann solution is a stationary $5 \mathrm{fth}$ contact wave. The Figure 2 shows that the stationary contact wave is well captured.

\subsection{Numerical test 2}

In this test, we consider the parameters (5.2). The approximate solution will be computed at the time $t=0.1$ on the interval $[-1,1]$ of the $x$-space with 10000 mesh points with C.F.L.=1/4. We consider the Riemann problem for (1.1)-(1.2) with the Riemann data given in Table 2.

\begin{tabular}{|c|c|c|}
\hline \multicolumn{3}{|l|}{ Table 2} \\
\hline Quantities \States & $U_{L}$ & $U_{R}$ \\
\hline$\alpha_{g}$ & 0.4 & 0.405 \\
\hline$p_{g}$ & 0.10800657 & 0.28752486 \\
\hline$u_{g}$ & -4.1912733 & -4.0012956 \\
\hline$p_{s}$ & 4.0582424 & 4.0906395 \\
\hline$u_{s}$ & 1.3809313 & 0.61850037 \\
\hline
\end{tabular}

The states that constitute the Riemann solutions are given in Table 3 .

\begin{tabular}{|c|c|c|c|c|}
\hline Table 3 & \multicolumn{5}{|l}{} \\
\hline$\searrow$ & $U_{1}$ & $U_{2}$ & $U_{3}$ & $U_{4}$ \\
\hline$\alpha_{g}$ & 0.4 & 0.4 & 0.4 & 0.405 \\
$p_{g}$ & 0.14761286 & 0.29267523 & 0.29267523 & 0.28752486 \\
$u_{g}$ & -8.12333333 & -4 & -4 & -4.0012956 \\
$p_{s}$ & 4.0582424 & 4.0582424 & 5.7995461 & 5.8458441 \\
$u_{s}$ & 1.3809313 & 1.3809313 & 1 & 1 \\
\hline
\end{tabular}

The Riemann solution begins with a 1 -shock wave from $U_{L}$ to $U_{1}$. This shock is followed by a 2-rarefaction wave from $U_{1}$ to $U_{2}$, followed by a 3 -shock wave from $U_{2}$ to $U_{3}$, followed by a contact discontinuity from $U_{3}$ to $U_{4}$ and then followed by a 4-shock wave from $U_{4}$ to $U_{R}$. The structure of the Riemann solution is shown in Figure 3 (upper-left corner).

The approximate solution and the exact solution are computed and displayed in Figure 3, where one can see that the approximate solution is very closed to the exact solution. This test indicates that the three-stage Roe-type scheme (4.11) can give a very desirable approximation to the exact solution.

\subsection{Numerical test 3}

In this test, we still take the parameters (5.2). The approximate solution is computed at the time $t=0.1$ on the interval $[-1,1]$ of the $x$-space with 10000 mesh points with C.F.L.=1/4. We consider the Riemann problem for (1.1)-(1.2) with the Riemann data given in Table 4. 

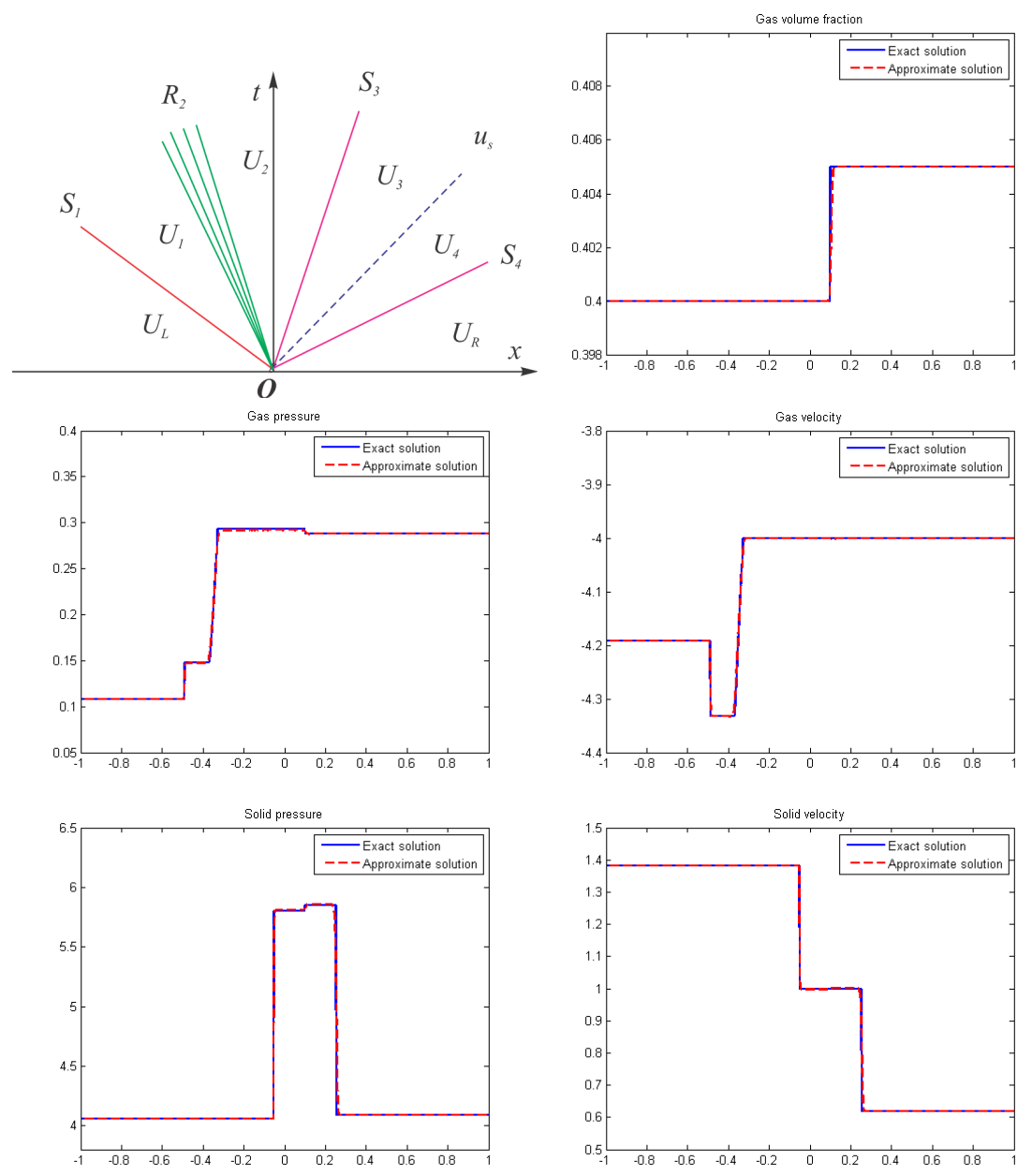

Figure 3. Test 2: The exact solution in the $(x, t)$-plane (upper-left corner), and approximations of the exact solution of the Riemann problem at the time $t=0.1$ in the interval $x \in[-1,1]$ with 10000 mesh points

Table 4

\begin{tabular}{c|c|c}
\hline Quantities \States & $U_{L}$ & $U_{R}$ \\
\hline$\alpha_{g}$ & 0.5 & 0.52 \\
$p_{g}$ & 3.5958182 & 6.818793 \\
$u_{g}$ & 0.034396019 & 0.067696575 \\
$p_{s}$ & 4.0582424 & 20.161735 \\
$u_{s}$ & 2.8346697 & -0.31449629 \\
\hline
\end{tabular}


The states that constitute the Riemann solutions are given in Table 5.

\begin{tabular}{|c|c|c|c|c|}
\hline Table 5 & \multicolumn{5}{|c|}{} \\
\hline$\searrow$ & $U_{1}$ & $U_{2}$ & $U_{3}$ & $U_{4}$ \\
\hline$\alpha_{g}$ & 0.5 & 0.5 & 0.52 & 0.52 \\
$p_{g}$ & 4.914414 & 4.914414 & 4.9560718 & 6.818793 \\
$u_{g}$ & -0.2 & -0.2 & -0.18230343 & 0.067696575 \\
$p_{s}$ & 4.0582424 & 27.857618 & 28.812697 & 28.812697 \\
$u_{s}$ & 2.8346697 & 0.2 & 0.2 & 0.2 \\
\hline
\end{tabular}

First, the Riemann solution starts by a 1 -shock wave from $U_{L}$ to $U_{1}$. The solution continues with a 3 -shock wave from $U_{1}$ to $U_{2}$, followed by a contact wave from $U_{2}$ to $U_{3}$, followed by a 2-rarefaction wave from $U_{3}$ to $U_{4}$ and then followed by a 4 -shock wave from $U_{4}$ to $U_{R}$. The Riemann solution has the structure as shown in Figure 4 (upper-left corner).

The approximate solution by the three-stage Roe-type scheme (4.11) is compared with the exact solution in Figure 4. This test shows that the scheme can give very good approximation to the exact solution.

\subsection{Numerical test 4}

In this test, the approximate solution will be computed at the time $t=0.1$ on the interval $[-1,1]$ of the $x$-space with 5000 mesh points with C.F.L. $=1 / 4$. The adiabatic exponents $\gamma_{k}, k=g, s$ are taken to be the ones of the air and water at $100^{\circ} \mathrm{C}$, see [35], for example. Precisely, the parameters are

$$
\gamma_{g}=1.4, \quad \gamma_{s}=1.324, \quad \kappa_{g}=1, \quad \kappa_{s}=2 .
$$

We consider the Riemann problem for (1.1)-(1.2) with the initial data given in Table 6

\begin{tabular}{|c|c|c|}
\hline \multicolumn{3}{|l|}{ Table 6} \\
\hline Quantities \States & $U_{L}$ & $U_{R}$ \\
\hline$\alpha_{g}$ & 0.5 & 0.505 \\
\hline$p_{g}$ & 0.15992239 & 0.1239783 \\
\hline$u_{g}$ & -1.1029741 & -1.4592393 \\
\hline$p_{s}$ & 6.7282569 & 8.6507854 \\
\hline$u_{s}$ & 0.42243168 & -2.0519671 \\
\hline
\end{tabular}

\begin{tabular}{|c|c|c|c|c|}
\hline Table 7 & \multicolumn{5}{|l|}{} \\
\hline$\searrow$ & $U_{1}$ & $U_{2}$ & $U_{3}$ & $U_{4}$ \\
\hline$\alpha_{g}$ & 0.5 & 0.5 & 0.505 & 0.505 \\
$p_{g}$ & 0.15992239 & 0.18534026 & 0.18546412 & 0.1239783 \\
$u_{g}$ & -1.1029741 & -1.2 & -1.1979253 & -1.4592393 \\
$p_{s}$ & 16.844816 & 16.844816 & 17.013093 & 17.013093 \\
$u_{s}$ & -1 & -1 & -1 & -1 \\
\hline
\end{tabular}



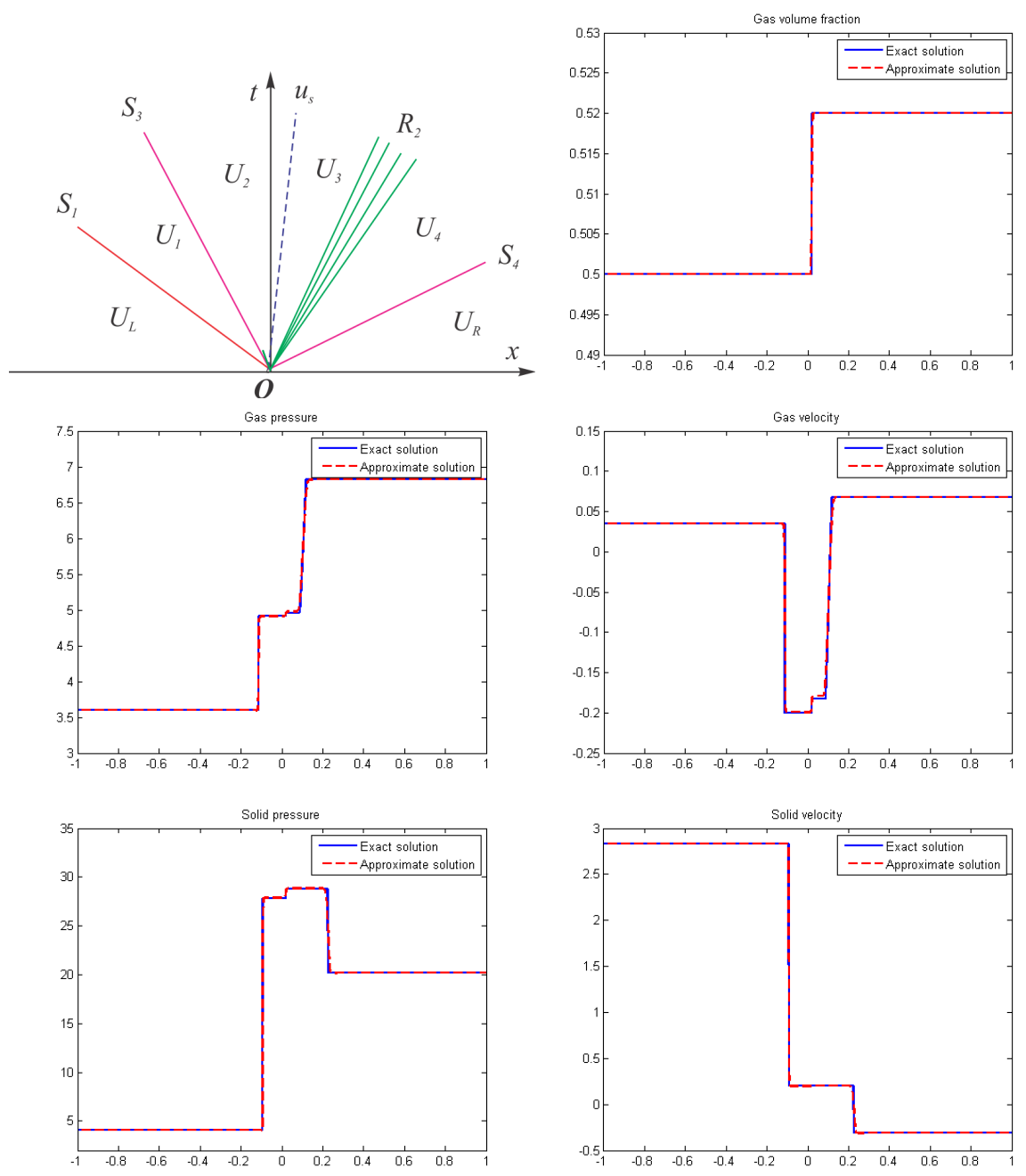

FiguRE 4. Test 3: The exact solution in the $(x, t)$-plane (upper-left corner), and approximations of the exact solution of the Riemann problem at the time $t=0.1$ in the interval $x \in[-1,1]$ with 10000 mesh points

The exact solution of the Riemann problem is a 3 -shock from $U_{L}$ to $U_{1}$, followed by a 1-shock from $U_{1}$ to $U_{2}$, a contact discontinuity from $U_{2}$ to $U_{3}$, a 2-shock from $U_{3}$ to $U_{4}$, and finally a 4 -shock from $U_{4}$ to $U_{R}$. The states that constitute the Riemann solutions are given in Table 7 . The structure of the Riemann solution is given by Figure 5 (upper-left corner). 

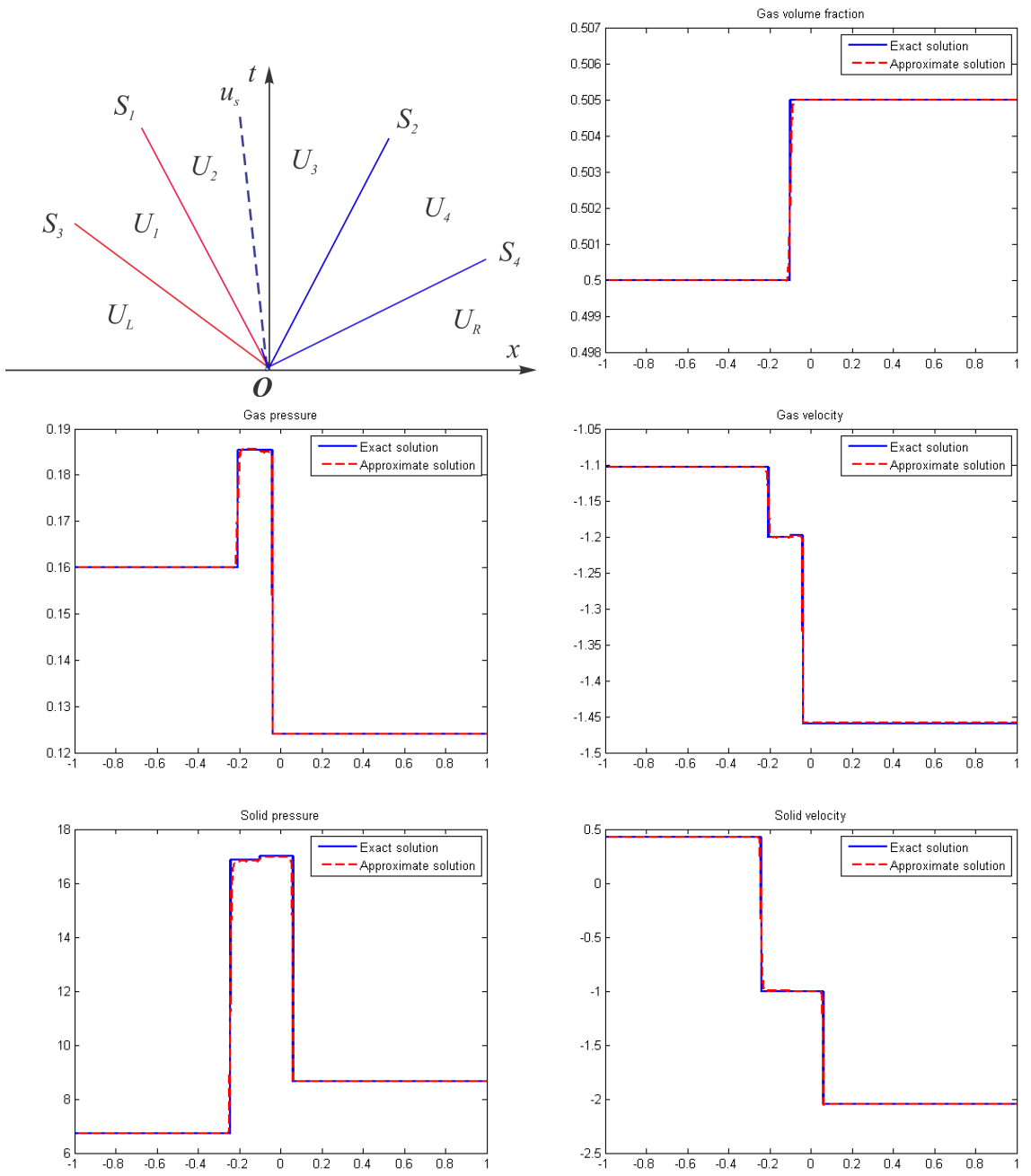

Figure 5. Test 4: The exact solution in the $(x, t)$-plane (upper-left corner), and approximations of the exact solution of the Riemann problem at the time $t=0.1$ in the interval $x \in[-1,1]$ with 5000 mesh points

The approximate solution by the three-stage Roe-type scheme (4.11) is compared with the exact solution in Figure 5. Again, this test shows that the scheme can give desirable approximations to the exact solution. 


\section{Concluding remarks}

Roe matrices are widely used in approximating solutions of hyperbolic systems of conservation laws, especially in gas dynamics equations. However, multi-phase flow models are often given as a system of balance laws in nonconservative form. Nonconservative terms in these models cause lots of inconvenience for standard numerical schemes. Although the two-phase flow model (1.1) is nonconservative, it is hyperbolic. We therefore develop in this paper the Roe-matrix technique to construct a numerical scheme (4.11) for the two-phase flow model (1.1). The technique consists of several stages, however, and the Roe-type scheme (4.11) is therefore "indirect" in some sense, where the Roe matrix is applied on intermediate states, rather than the "primary" states as in the original Roe scheme versions. The scheme is shown to be well-balanced, and provides us with good approximations to the exact solutions.

\section{References}

[1] A. Ambroso, C. Chalons, F. Coquel, and T. Galié, Relaxation and numerical approximation of a two-fluid two-pressure diphasic model, Math. Model. Numer. Anal. 43 (2009), no. 6, 1063-1097.

[2] N. Andrianov and G. Warnecke, The Riemann problem for the Baer-Nunziato two-phase flow model, J. Comput. Phys. 195 (2004), no. 2, 434-464.

[3] E. Audusse, F. Bouchut, M.-O. Bristeau, R. Klein, and B. Perthame, A fast and stable well-balanced scheme with hydrostatic reconstruction for shallow water flows, SIAM J. Sci. Comput. 25 (2004), no. 6, 2050-2065.

[4] M. R. Baer and J. W. Nunziato, A two-phase mixture theory for the deflagration-todetonation transition (ddt) in reactive granular materials, Int. J. Multiphase Flow 12 (1986), no. 6, 861-889.

[5] R. Botchorishvili, B. Perthame, and A. Vasseur, Equilibrium schemes for scalar conservation laws with stiff sources, Math. Compu. 72 (2003), no. 241, 131-157.

[6] R. Botchorishvili and O. Pironneau, Finite volume schemes with equilibrium type discretization of source terms for scalar conservation laws, J. Comput. Phys. 187 (2003), no. 2, 391-427.

[7] J. B. Bzil, R. Menikoff, S. F. Son, A. K. Kapila, and D. S. Steward, Two-phase modelling of a deflagration-to-detonation transition in granular materials: A critical examination of modelling issues, Phys. Fluids 11 (1999), no. 2, 378-402.

[8] A. Chinnayya, A.-Y. LeRoux, and N. Seguin, A well-balanced numerical scheme for the approximation of the shallow water equations with topography: the resonance phenomenon, Int. J. Finite Vol. 1 (2004), no. 1, 33 pp.

[9] G. Dal Maso, P. G. LeFloch, and F. Murat, Definition and weak stability of nonconservative products, J. Math. Pures Appl. (9) 74 (1995), no. 6, 483-548.

[10] P. Embid and M. Baer, Mathematical analysis of a two-phase continuum mixture theory, Contin. Mech. Thermodyn. 4 (1992), no. 4, 279-312.

[11] T. Gallouët, J.-M. Hérard, and N. Seguin, Numerical modeling of two-phase flows using the two-fluid two-pressure approach, Math. Models Methods Appl. Sci. 14 (2004), no. $5,663-700$.

[12] P. Goatin and P. G. LeFloch, The Riemann problem for a class of resonant hyperbolic systems of balance laws, Ann. Inst. H. Poincaré Anal. Non Linéaire 21 (2004), no. 6881902. 
[13] J. M. Greenberg and A. Y. Leroux, A well-balanced scheme for the numerical processing of source terms in hyperbolic equations, SIAM J. Numer. Anal. 33 (1996), no. 1, 1-16.

[14] J. M. Greenberg, A. Y. Leroux, R. Baraille, and A. Noussair, Analysis and approximation of conservation laws with source terms, SIAM J. Numer. Anal. 34 (1997), no. 5, 1980-2007.

[15] S. Jin and X. Wen, An efficient method for computing hyperbolic systems with geometrical source terms having concentrations, J. Comput. Math. 22 (2004), no. 2, 230-249.

[16] S. Karni and G. Hernández-Duenas, A hybrid algorithm for the Baer-Nunziato model using the Riemann invariants, J. Sci. Comput. 45 (2010), no. 1-3, 382-403.

[17] B. L. Keyfitz, R. Sander, and M. Sever, Lack of hyperbolicity in the two-fluid model for two-phase incompressible flow, Discrete Contin. Dyn. Syst. Ser. B 3 (2003), no. 4, $541-563$.

[18] D. Kröner, P. G. LeFloch, and M. D. Thanh, The minimum entropy principle for compressible fluid flows in a nozzle with discontinuous cross-section, Math. Model. Numer. Anal. 42 (2008), no. 3, 425-442.

[19] D. Kröner and M. D. Thanh, Numerical solutions to compressible flows in a nozzle with variable cross-section, SIAM J. Numer. Anal. 43 (2005), no. 2, 796-824.

[20] M.-H. Lallemand and R. Saurel, Pressure relaxation procedures for multiphase compressible flows, INRIA Report (2000), No. 4038.

[21] P. G. LeFloch and M. D. Thanh, The Riemann problem for fluid flows in a nozzle with discontinuous cross-section, Commun. Math. Sci. 1 (2003), no. 4, 763-797.

[22] _ . The Riemann problem for the shallow water equations with discontinuous topography, Commun. Math. Sci. 5 (2007), no. 4, 865-885.

[23] — A Godunov-type method for the shallow water equations with discontinuous topography in the resonant regime, J. Comput. Phys. 230 (2011), no. 20, 7631-7660

[24] S. T. Munkejord, Comparison of Roe-type methods for solving the two-fluid model with and without pressure relaxation, Computers \& Fluids 36 (2007), no. 6, 1061-1080.

[25] R. Saurel and R. Abgrall, A multiphase Godunov method for compressible multifluid and multiphase flows, J. Comput. Phys. 150 (1999), no. 2, 425-467.

[26] D. W. Schwendeman, C. W. Wahle, and A. K. Kapila, The Riemann problem and a high-resolution godunov method for a model of compressible two-phase flow, J. Comput. Phys. 212 (2006), no. 2, 490-526.

[27] M. D. Thanh, A phase decomposition approach and the Riemann problem for a model of two-phase flows, preprint.

[28] _ The Riemann problem for a nonisentropic fluid in a nozzle with discontinuous cross-sectional area, SIAM J. Appl. Math. 69 (2009), no. 6, 1501-1519.

[29] _ Exact solutions of a two-fluid model of two-phase compressible flows with gravity, Nonlinear Anal. Real World Appl. 13 (2012), no. 2, 987-998.

[30] _ On a two-fluid model of two-phase compressible flows and its numerical approximation, Commun. Nonlinear Sci. Numer. Simul. 17 (2012), no. 1, 195-211.

[31] M. D. Thanh and A. Izani Md. Ismail, A well-balanced scheme for a one-pressure model of two-phase flows, Phys. Scr. 79 (2009), no. 6, 065401, 7pp.

[32] M. D. Thanh, Md. Fazlul Karim, and A. Izani Md. Ismail, Well-balanced scheme for shallow water equations with arbitrary topography, Int. J. Dyn. Syst. Differ. Equ. 1 (2008), no. 3, 196-204.

[33] M. D. Thanh, D. Kröner, and C. Chalons, A robust numerical method for approximating solutions of a model of two-phase flows and its properties, Appl. Math. Comput. 219 (2012), no. 1, 320-344.

[34] M. D. Thanh, D. Kröner, and N. T. Nam, Numerical approximation for a Baer-Nunziato model of two-phase flows, Appl. Numer. Math. 61 (2011), no. 5, 702-721.

[35] F. M. White, Fluid Mechanics, 7th ed. McGraw-Hill, 2010. 
Department of Mathematics

INTERNATIONAL UNIVERSITY

Quarter 6, Linh Trung Ward

Thu Duc District, Ho Chi Minh City, Vietnam

E-mail address: mdthanh@hcmiu.edu.vn 